\title{
Free-Electron Laser Experiments With and Without a Guide Magnetic Field: A Review of Millimeter-Wave Free-Electron Laser Research at the Naval Research Laboratory
}

\author{
JOHN A. PASOUR AND STEVEN H. GOLD
}

(Invited Paper)

\begin{abstract}
A review of the millimeter-wave free-electron laser experimental program at the Naval Research Laboratory is presented. Specific attention is focused on the recent results of superradiant and amplifier experiments on a pulseline accelerator using an axial guide magnetic field, and on the results of a superradiant experiment on a linear induction accelerator without a guide magnetic field.
\end{abstract}

\section{INTRODUCTION}

$\mathrm{T}$ HE free-electron laser (FEL), when driven by an intense electron beam of moderate energy $(\sim 1 \mathrm{MeV})$ and low parallel velocity spread $\left(\Delta \gamma_{z} / \gamma_{z} \leqslant 1\right.$ percent), is capable of generating extremely high-power, continuously tunable millimeter wavelength radiation with high gain and moderate efficiency. Such a device may have important applications, either as an oscillator or an amplifier, for such purposes as plasma heating, advanced electron accelerators, communications, or for other high-power applications for which no comparable sources exist. For this reason, there has been great interest in investigating the physics of such devices and demonstrating their experimental feasibility.

The Naval Research Laboratory (NRL) has played a pioneering role in the experimental development of FEL's in this operating regime, as well as in developing the theory for such devices. The experimental work began in the early 1970's when high-current pulseline accelerators became available, and resulted from the desire to employ the intense relativistic electron beams made possible by these devices for high-power microwave generation. At that time Friedman and Herndon [1] used such an accelerator to generate powerful microwave emission. They employed a rippled-magnetic-field, fast-wave configuration similar to that used by R. M. Phillips in his earlier ubitron experiments [2], [3], in an attempt to scale the ubitron results to very high powers. It is now generally recognized that the ubitron mechanism was identical to what is now termed

Manuscript received November 29, 1984; revised February 12, 1985 This work was supported by the Office of Naval Research.

The authors are with the Plasma Physics Division, Naval Research Laboratory, Washington, DC 20375 . the FEL interaction, even though the electron beam energy was quite low, and that Phillips' series of experiments were the first serious investigation of long-wavelength FEL devices. Early FEL experiments using intense relativistic electron beams were frequently referred to as stimulated scattering [4], but after the infrared FEL experiments at Stanford University [5], [6] popularized the term "free-electron laser," and the equivalence of the FEL and stimulated scattering mechanisms was shown [7], [8], the high-current, millimeter-wave experiments also began to be referred to as FEL's.

The first intense relativistic electron beam experiments to be interpreted as stimulated scattering were performed at NRL beginning in 1972 [9], [10]. These experiments were designed to operate as cyclotron masers at a wavelength of $\sim 2 \mathrm{~cm}$, but st rong submillimeter-wavelength emission was also observed. It was postulated that this high frequency output was generated when the lower frequency maser radiation was reflected from the output window back onto the electron beam. This reflected radiation was then scattered from the beam, this time with a Doppler frequency upshift, to produce the submillimeter radiation. The low frequency radiation therefore became the electromagnetic wiggler for an FEL interaction. A followup experiment was performed in 1976 [11] that was designed to maximize the FEL interaction and unequivocally demonstrate this mechanism by separating the maser and FEL interaction regions. When the lengths of the two regions were optimized, $400 \mu \mathrm{m}$ radiation was generated at a power of $\sim 1 \mathrm{MW}$.

At about the same time this experiment was performed, a group at Columbia University carried out a series of experiments in which the electromagnetic wiggler was replaced by a periodic magnetic wiggler [12]-[14]. These experiments also produced $\sim 1 \mathrm{MW}$ of millimeter-wavelength radiation. The congruence of research interests at Columbia University and NRL led to a collaborative effort at NRL in 1978, in which experimental studies of FEL operation in both superradiant and oscillator configurations were performed [15]. The results showed a signifi- 
cant line narrowing, from $\sim 10$ to $\sim 2$ percent, when feedback was introduced into the experiment. Again, about $1 \mathrm{MW}$ of $400 \mu \mathrm{m}$ radiation was generated, and the wavelength agreed well with theory. However, although the very high powers produced in these early experiments were very encouraging, the efficiencies of all of them were quite low $(<0.2$ percent $)$.

When viewed in retrospect, it became apparent that the low efficiencies observed in the previous experiments were most likely the result of a variety of apparent experimental deficiencies, the most significant of which was inadequate electron beam quality. To address these issues, new experimental studies were undertaken at NRL. The primary focus of these studies was to demonstrate the efficient production of very high-power millimeter-wave FEL radiation using intense relativistic electron beams with kinetic energies of order $1 \mathrm{MeV}(\gamma \sim 3)$, and to investigate the physics of such devices.

In any FEL device, the principal factors in determining the overall operating characteristics are the macroscopic and microscopic parameters of the electron beam used to drive the interaction. These beam parameters combine with the parameters of the wiggler magnetic field to define the operating range of the device. The primary determinant of the macroscopic parameters (beam current, current density, and energy) is the technology used to generate the accelerating voltage pulse. The microscopic characteristics (emittance, energy spread) are determined by the diode design and the beam optics. Two separate technologies, a conventional pulseline accelerator and a linear induction accelerator, have been employed in these recent studies. The characteristics of the beams produced. by each of these accelerators have a great bearing on the remaining details of the two experiments.

One NRL group began a program to generate much higher quality electron beams using a pulseline accelerator, and to study the physics of the FEL interaction using such beams. One of the experiments reviewed in this paper is a direct outgrowth of that effort, and employs the same pulseline accelerator (VEBA) that was used in the earlier NRL experiments. However, significant modifications have been made in the electron beam diode, to greatly improve the beam quality, as well as in other critical experimental details. The other experiment that is discussed below employs a linear induction accelerator (LIA) which has a much longer pulse duration but a somewhat smaller current. These experiments will be dealt with in separate sections below.

\section{Pulseline Accelerator Experiments}

\section{A. Background}

The experiments on the VEBA pulseline accelerator make use of a conventional pulseline accelerator operating at beam kinetic energies of $0.9-1.35 \mathrm{MeV}$ with a $50 \mathrm{~ns}$ voltage pulse. The basic experimental configuration is shown in Fig. 1. A $6 \mathrm{~mm}$ diameter solid electron beam of 0.6-1.5 kA with a low parallel velocity spread traverses a

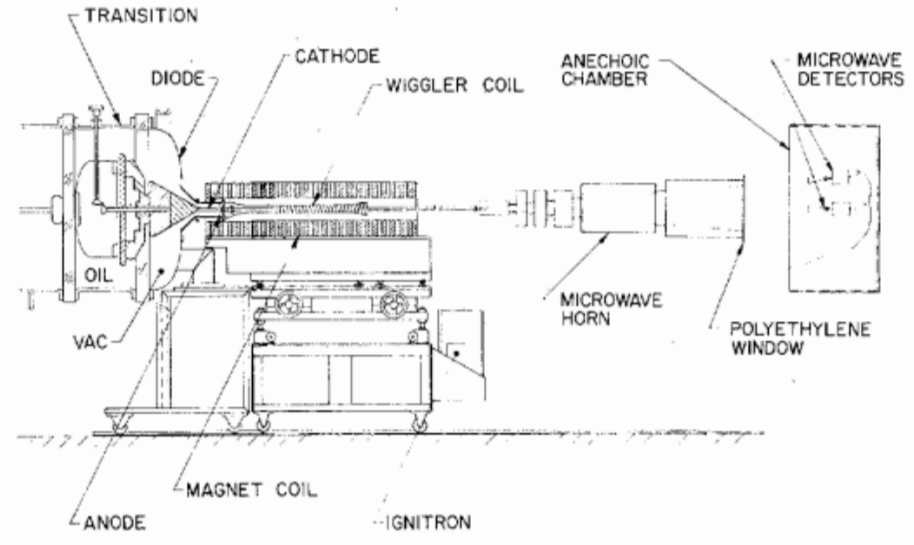

Fig. 1. Scale drawing of the VEBA FEL experiment, from the front end of the accelerator to the microwave detectors.

$10.8 \mathrm{~mm}$ i.d. stainless-steel drift tube under the combined influence of an axial guide magnetic field and a transverse wiggler magnetic field. At the end of the interaction region, the beam is allowed to expand into the wall of the drift tube, and the millimeter-wave radiation is typically radiated into the laboratory through a large microwave horn consisting of a $1.8 \mathrm{~m}$ long conical taper up to a 30 $\mathrm{cm}$ i.d. polyethylene vacuum window.

The electron beam is produced by aperturing the emission of a special computer-optimized field-emission diode in order to scrape $>90$ percent of the total diode current. (This has an effect similar to the "emittance filtering" that has been employed on other types of accelerators, such as linear induction accelerators, in order to make beams suitable for FEL experimentation [16].) While the beam is produced in an inefficient manner, and uses a technology (plasma-induced field emission) that is generally suitable only for short pulse $(<<1 \mu$ s) operation, the resulting electron beam has a current density of $\sim 2-5 \mathrm{kA} / \mathrm{cm}^{2}$ and a low velocity spread $\left(\Delta \gamma_{z} / \gamma_{z}<1\right.$ percent, where $\gamma_{z}$ is the relativistic factor calculated from the beam axial velocity) that is suitable for FEL experimentation in either the collective or strong-pump regimes [8] in the millimeter wavelengths.

The $3 \mathrm{~cm}$ periodicity wiggler magnetic field is produced by pulsing a capacitor bank through a pair of bifilar windings on a $1 \mathrm{~m}$ coil form that is flared at either end. The result is a circularly polarized pump field that increases gradually over seven wiggler periods, to provide an adiabatic entry into the wiggler, is uniform over a 21 period long interaction region, and decreases to zero over an additional five periods, so that the electrons leave the wiggler without large amounts of transverse kinetic energy.

As a result of the very high current densities employed, the beam is produced with and must remain confined by an axial magnetic field. Furthermore, the electron beam quality is a function of the magnitude of the axial magnetic field, and for best operation, an axial magnetic field in excess of $10 \mathrm{kG}$ must be employed. The presence of this axial field significantly affects the FEL interaction in several fundamental ways. First, the electron trajectories are strongly modified by the presence of an axial magnetic 


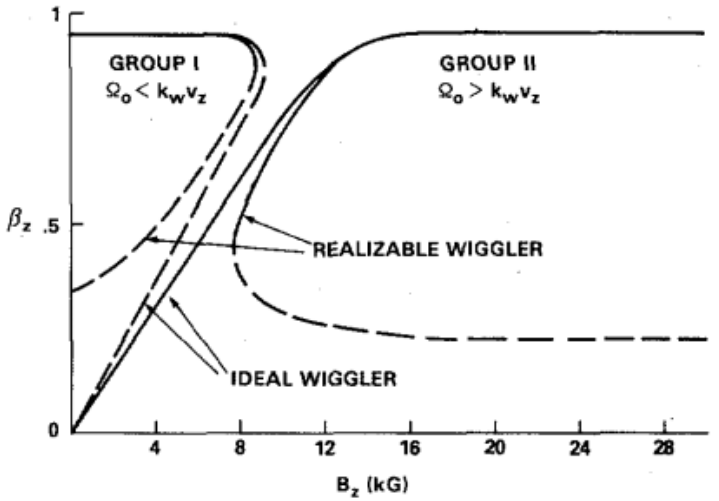

(a)

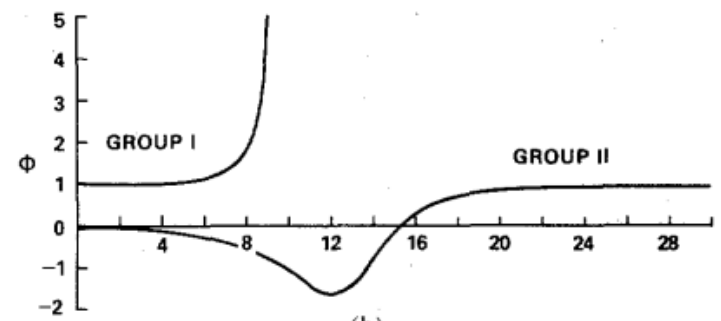

(b)

Fig. 2. Plots of (a) $\beta$, and (b) $\Phi$ versus axial guide field for $B_{r}=630 \mathrm{G}$ and $\gamma=3.4$. The axial velocity is shown both for an ideal wiggler, and for axicentered orbits in a realizable wiggler. The dashed lines describe unstable orbits. Here $\Phi$ is calculated for the ideal wiggler.

field, particularly when the wiggler-induced helical motion is close to resonance with the cyclotron period in the axial magnetic field. This gyroresonant effect strengthens the FEL interaction by increasing the amplitude of the helical motion driven by the wiggler field. Second, in addition to its effects on the particle motion, the axial magnetic field directly affects the strength of the FEL coupling through its effect on the ponderomotive potential, and can change the nature of the FEL instability. Third, the axial magnetic field makes possible an additional instability mechanism, the cyclot ron maser instability, which can play a role in these experiments.

From the equations of motion in the presence of both axial and ideal helical wiggler magnetic fields, the electron velocity can be partitioned into axial and perpendicular components along ideal helical trajectories [17]:

$$
\begin{aligned}
\beta_{\perp} & =\beta_{z} \Omega_{r} /\left(\Omega_{0}-c \beta_{z} k_{w}\right) \\
\beta_{z} & =\left(1-\gamma^{-2}-\beta_{\perp}^{2}\right)^{1 / 2} .
\end{aligned}
$$

Here, $\beta_{\perp, z} \equiv v_{\perp, z} / c, c$ is the velocity of light, $\Omega_{0, r} \equiv$ $\left|e B_{z, r} / \gamma m c\right|$ are the relativistic cyclotron frequencies based on the axial and wiggler magnetic fields, $m$ and $e$ are the electron rest mass and charge, $\gamma$ is the relativistic mass factor, and $k_{w} \equiv 2 \pi / \lambda_{w}$, where $\lambda_{w}$ is the wavelength of the wiggler magnetic field. For the proper sense of the axial magnetic field, these equations introduce a gyroresonance into the electron motion, as seen in Fig. 2(a) for parameters typical of the superradiant FEL experiments on the VEBA accelerator. Note that the "ideal wiggler" curve in this figure corresponds to the use of a one-dimensional wiggler theory, in which the wiggler field is considered to be purely transverse and without radial variation.

A three-dimensional orbit theory exists [18], which takes into account both the radial variation of the transverse wiggler field amplitude, as well as the axial component of the wiggler field. A calculation based on this theory is shown in the figure as the line labeled "reafizable wiggler." However, this theory applies only to axicentered orbits in the "realizable wiggler," in which the electrons are assumed to move along a particular helical trajectory in a constant phase relationship with the wiggler field. The electrons then experience both axial and radial wiggler field components which are determined by the self-consistent orbit radius and azimuthal phase, but which are constant along the orbit. This is not an accurate model to use for calculations involving a finite-radius solid electron beam, in which the electrons will execute approximately helical trajectories about their initial radii, rather than about the axis of the wiggler magnetic field, and thus will not remain in a constant phase relationship with the components of the wiggler field. For that reason, the one-dimensional orbit theory is used in this paper, but the specified wiggler field employed in the calculations is an average over the beam radius, rather than simply the value of the transverse wiggler field on axis.

The axial magnetic field affects the strength of the FEL interaction through the coupling parameter

$$
\Phi=1-\beta_{w}^{2} \gamma_{z}^{2} \Omega_{0} /\left[\left(1+\beta_{w}^{2}\right) \Omega_{0}-k_{w} v_{z}\right]
$$

which appears in the FEL dispersion equations [19]. Here, $\beta_{w}=\beta_{\perp} / \beta_{z}$, and $\gamma_{z}=\left(1-\beta_{z}^{2}\right)^{-1 / 2}$. While $\Phi$ reduces to unity if either $B_{z}$ or $B_{r}$ vanishes, the combination of nonzero axial and wiggler fields results in significant variations in the value of $\Phi$, which results in substantial changes in the effective beam plasma frequency as well as in the strength of the FEL instability. Variation of $\Phi$ as a function of $B_{z}$ is shown in Fig. 2(b) for some typical experimental parameters. $\Phi$ can be either positive or negative, and its sign significantly affects the nature of the FEL instability. For $\Phi>0$, one recovers the usual FEL regime, in which the collective FEL instability couples a stable electromagnetic wave with a stable beam space charge mode. Instability (and amplification) results when the positive energy electromagnetic wave is resonant with the pumpshifted negative energy space-charge wave on the beam. However, the regime above gyroresonance in which $\Phi$ is negative is particularly interesting because the FEL interaction then couples the electromagnetic mode to a beam space-charge wave, which is itself driven unstable by the presence of the wiggler magnetic field [20]. This regime is predicted to offer broader bandwidth as well as higher efficiency [21], and in fact is the regime in which the experiment is observed to operate best. For these reasons, an axial magnetic field is an attractive feature that may be incorporated in future devices that do not require it solely for reasons of beam formation and space charge control.

However, the presence of an axial magnetic field is also 
a significant experimental complication, since it makes possible an additional instability mechanism capable of generating electromagnetic radiation, a relativistic negative-mass instability known as the cyclotron maser instability [22]. Typically, a large variety of such interactions are possible, in which the first or higher harmonics of the cyclotron frequency are coupled to a variety of modes of the interaction waveguide. Furthermore, there are also possible couplings involving both a cyclotron harmonic and the wiggler period [23]. Thus, the mere presence of a wiggler does not guarantee that the FEL mechanism will be dominant in a particular device, and counterexamples exist in the literature (see, e.g., [24]). While the cyclotron maser and FEL mechanisms may both be attractive for very high-power millimeter-wave generation [25], there is always a strong experimental interest in understanding the operative mechanism in an experiment. For this reason, care must be taken to distinguish experimentally between the FEL and cyclotron maser processes in any experiment utilizing both axial and wiggler magnetic fields.

On the other hand, it is possible to design an experiment in which the FEL mechanism should be dominant. To begin with, ideal helical motion, generated by the adiabatic entry of a paraxial electron beam into a region of helical wiggler magnetic field while under the influence of a uniform axial magnetic field, is tied to the spatial phase of the wiggler field, and is not subject to the usual cyclotronmaser azimuthal bunching instability mechanism. On the other hand, any additional transverse electron kinetic energy in Larmor motion, due to transverse beam energy produced either in the diode or generated by a nonideal entry into the region of uniform wiggler field, can couple to the cyclotron maser mechanism. The cyclotron maser instability is thus driven by a separate fraction of the electron motion than the FEL instability, and the two instabilities appear to be linearly independent. (This distinction begins to break down very near to gyroresonance.) Either instability may grow, depending on the microscopic character of the electron motion in the interaction region. However, based on some recent calculations in the parameter range of these experiments, the FEL growth rate for a cold electron beam appears theoretically to be somewhat larger than the cyclotron maser growth rate for a similar beam, based on equal amplitudes of transverse velocity either of axicentered wiggler-induced helical motion or of axicentered Larmor motion in the absence of a wiggler [26]. (The FEL and cyclotron maser instabilities are typically analyzed theoretically in isolation from each other, and often by different researchers, so that a general theory incorporating both mechanisms in the presence of both axial and wiggler magnetic fields remains to be produced.) Furthermore, in a well-designed experiment, the wiggler motion should predominate over Larmor motion, further enchancing the growth rate of the FEL instability relative to the cyclotron maser mechanism. Therefore, the FEL mechanism can be expected to have a substantially higher relative growth rate, so that in a high gain experiment, FEL emission is likely to dominate the emission spectrum.
From the viewpoint of experimental diagnostics, microwave radiation emerging from an experiment is characterized by power, frequency spectrum, polarization, and waveguide mode, but it does not directly carry any additional memory of the instability mechanism that caused its generation or amplification. Furthermore, the detailed knowledge of the electron phase space needed to predict the growth rate of the cyclotron maser mechanism is not generally experimentally accessible. Thus, the experimental distinction between the two instability mechanisms can only be made by a detailed comparison of the experimental behavior with predictions of frequency, mode, and power scaling derived from either model. Depending on the experimental parameters, in particular, on how close operation is to gyroresonance, and on the error bars on these experimental parameters, this distinction may be either clearcut or ambiguous.

It is precisely near gyroresonance that the electron motion is expected to depart most substantially from the ideal helical motion that is desired in an FEL, and thus, potentially, to couple most strongly to the cyclotron maser instability; this is due in part to the difficulty of adiabatic entry into the region of uniform wiggler fields when operating near gyroresonance. For this reason, the portion of parameter space very near to gyroresonance that is most ambiguous, in terms of distinguishing the two instability mechanisms, is avoided experimentally. The detailed frequency and power scaling observed in these experiments is strong evidence that FEL emission is the dominant mechanism of radiation production. In addition, the closer the experimental parameters approach gyroresonance at constant (calculated) helical $\beta_{\perp}$, the lower the saturated emission that has been experimentally observed in these FEL experiments.

Two classes of FEL experiments have been carried out on the VEBA accelerator, superradiant experiments and true amplifier experiments. In the superradiant experiments, spontaneous emission from the electron beam due to the presence of the wiggler field is highly amplified by the FEL interaction in a single pass through the interaction region, resulting typically in tens of megawatts of millimeter-wave emission that grows to saturation, in essence, from amplifier noise. Temporal isolation is used to avoid feedback effects during the short accelerator voltage pulse, in order to produce an unambiguous single pass result. In the true amplifier experiments, a signal wave substantially larger than the noise spectrum is injected into the FEL interaction region and coherently amplified to very high power in a single pass through the interaction region. For the parameters of the amplifier experiment, superradiant saturation will not occur without incorporating intentional feedback into the experiment. The superradiant experiments have the virtue of imposing no frequency constraints on the interaction, so that the final emission spectrum is the result of linear growth and possible saturation of the instability over the entire instantaneous bandwidth of the FEL interaction. For that reason, as well as for reasons of experimental simplicity, the first 
experiments to be carried out were in the superradiant configuration.

\section{B. Superradiant Experiments}

The first series of experiments was completed in 1981 and reported by R. K. Parker et al. [27]. Operating at 1.35 $\mathrm{MeV}$ and $1 \mathrm{kA}$ in a superradiant configuration, these experiments produced $35 \mathrm{MW}$ of radiation at approximately $75 \mathrm{GHz}$ with an interaction efficiency of 2.5 percent. This power level, which was determined calorimetrically, was a record at this frequency for any coherent device and an improvement in experimental efficiency by an order of magnitude over all previous FEL experiments using intense relativistic electron beams. This improvement in performance was attributed to the care taken with the quality of the electron beam used in the experiments, which permitted operation in the collective regime. These results made it apparent that the very low efficiencies seen in the previous long wavelength FEL experiments using intense relativistic electron beams were most likely due to the use of electron beams that did not satisfy the axialvelocity-spread constraints for a collective FEL interaction. Also reported was the discovery of a very simple scaling relationship between the amplitude of the FEL emission and the calculated magnitude of $\beta_{\perp}$ produced by various combinations of $B_{r}$ and $B_{z}$ at constant $\gamma$, with only a weak additional dependence on the magnitude of $\boldsymbol{B}_{2}$. This scaling relationship was found to be true both above and below gyroresonance. The observation of this simple scaling of emission with $\beta_{\perp}$ was the first experimental demonstration that the simple single particle picture of electron trajectories, shown in Fig. 2, applied to an experiment using an intense relativistic electron beam, and it was a clear indication that FEL emission was likely to be the dominant experimental effect. These results were expanded upon in a paper by S. H. Gold et al. [28]. A subsequent paper by R. H. Jackson et al. [29] spelled out the details of the diode design, carried out computational and experimental studies of beam quality, and verified that the beam propagation through the wiggler was in good agreement with calculations based on the single particle orbit theory. It reported measurements of radiation onsets and cutoffs as a function of experimental parameters, both above and below gyroresonance, that agreed with predictions based on single-particle orbits and on a recently developed coldbeam Raman FEL theory [19]. It also carried out preliminary studies of emission wavelength, based on the use of high pass and mode filters, that identified the radiation as FEL emission.

These results were noted by other workers, and have resulted in a variety of related experimental work. The concern for beam temperature led a group at Columbia Univeristy to construct a Thomson backscattering diagnostic for electron beam parallel velocity spread, in order to determine the characteristics of electron beams for use in their future FEL experiments. They performed measurements on a close copy of the VEBA FEL diode, and

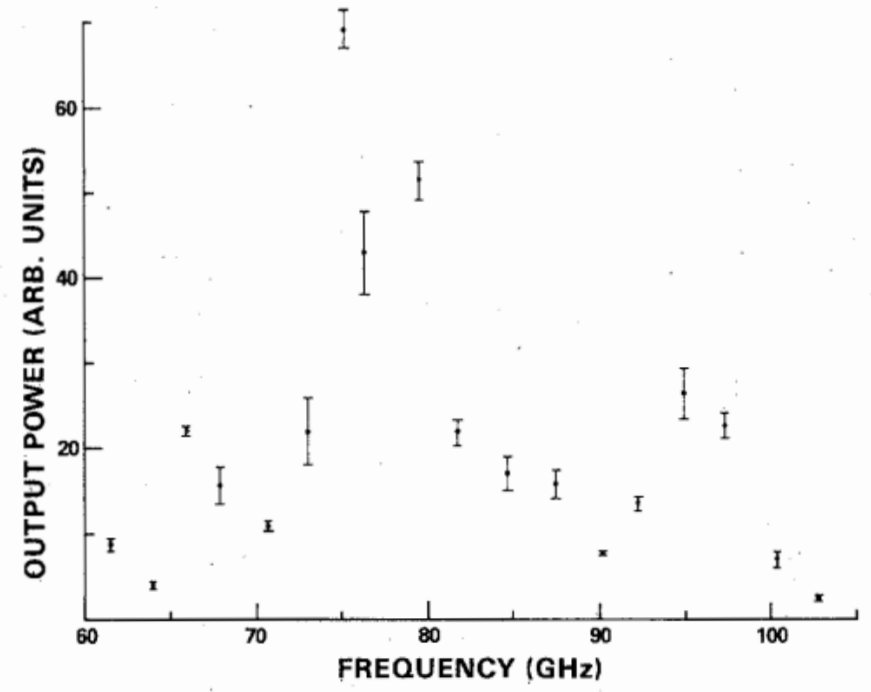

Fig. 3. Free-electron laser emission spectrum for $\gamma=3.4, B_{z}=16 \mathrm{kG}$, $B_{r}=1.4 \mathrm{kG}, \beta_{\perp} \sim 0.265$. Coupling is predicted to the $\mathrm{TE}_{\mathrm{It}}$ mode at $\sim 97 \mathrm{GHz}$ and the $\mathrm{TM}_{01}$ mode at $\sim 80 \mathrm{GHz}$. Error bars at each frequency are statistical. The estimated systematic error is $\pm 2 \mathrm{~dB}$.

reported that the claims made for the low-velocity-spread beam were in fact valid [30]. A group at the Laboratoire de Physique des Milieux Ionisés of the Ecole Polytech-. nique under J. M. Buzzi, whose earlier FEL experiments produced significant radiation, at low efficiency and with a very broad-band bandwidth, only very close to gyroresonance [31], has also assembled a Thomson scattering diagnostic for the same purpose [32]. Various groups have constructed new experiments in which great care is taken with electron beam quality (see, e.g., [33]-[35]).

The results of further experiments, studying the details of the emission spectrum, the spatial growth rate of the spontaneous emission, efficiency enhancement, and the production of air plasmas using the intense focused millimeter-wave emission from the experiment, were reported in a series of four additional experimental publications by S. H. Gold et al. [36]-[39]. These experiments focused on operation above gyroresonance where $\Phi<0$, the regime in which the earlier work had demonstrated the best operation. In these experiments, the efficiency was further improved to 6 percent, resulting in the production of $75 \mathrm{MW}$ of radiation at frequencies near $75 \mathrm{GHz}$, as confirmed calorimetrically. Emission spectra were observed, using a millimeter-wave quasi-optical grating spectrometer [40], that generally agreed with predictions for the FEL interaction. Coupling was observed to the two lowest order modes, the $\mathrm{TE}_{11}$ and $\mathrm{TM}_{01}$ modes, of the cylindrical stainless-steel interaction waveguide, as illustrated in Fig. 3. These observed spectral peaks are in reasonable agreement with the predicted FEL coupling frequencies shown in Fig. 4, and allow a distinction to be made with respect to predictions for the cyclotron maser mechanism. The distinction is most clear cut far from gyroresonance, as is the case in Fig. 3, since the comparable cyclotron maser couplings to these two modes would occur some $60-70 \mathrm{GHz}$ higher in frequency.

Tuning of the emission spectrum was demonstrated by 


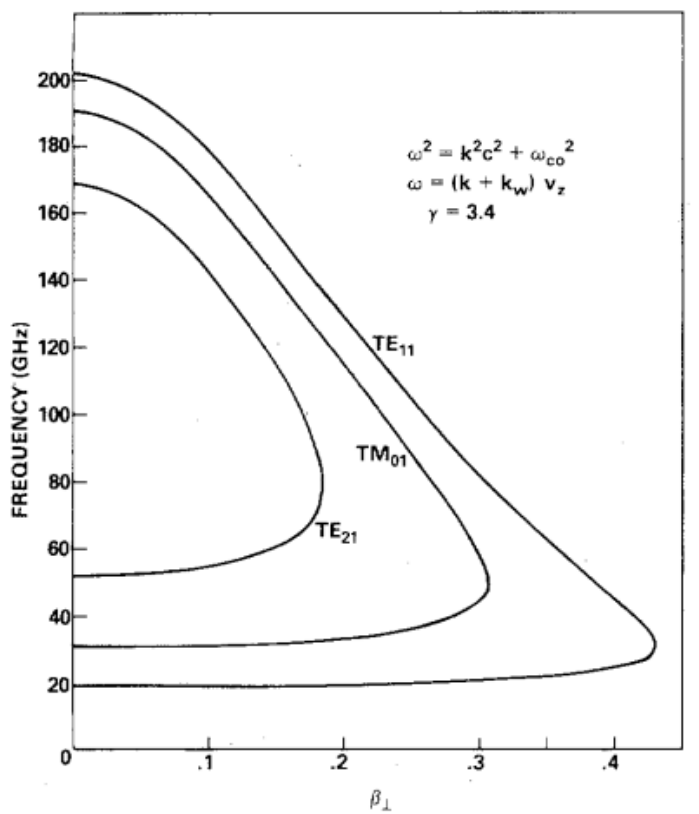

Fig. 4. Predicted FEL coupling frequencies as a function of $\beta_{\perp}$ for $\gamma=$ 3.4 , calculated from the crossing intersections for the pump-shifted beam line, $\omega=\left(k+k_{w}\right) v_{\varepsilon}$, with the dispersion relation for the electromagnetic mode in the empty waveguide.

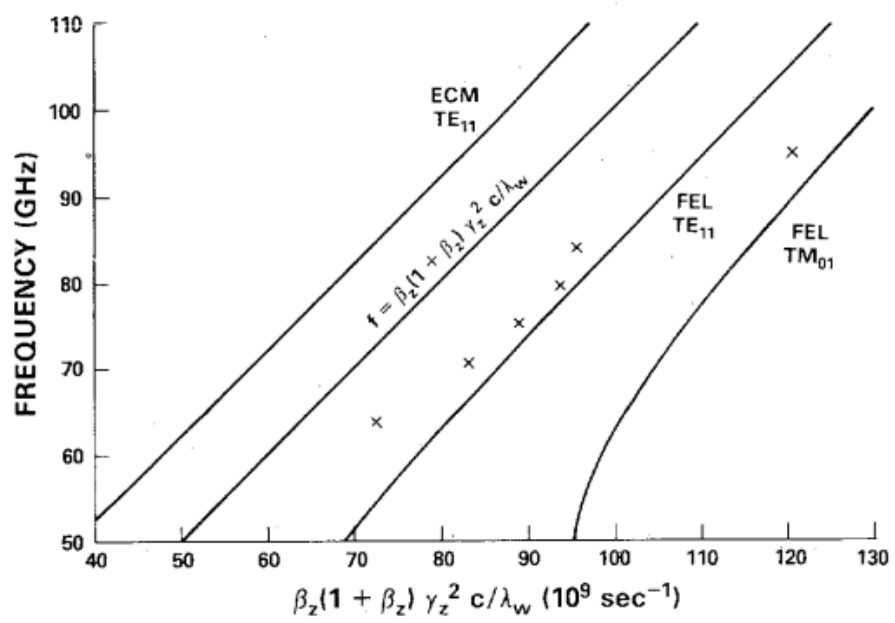

Fig. 5. Emission frequencies plotted against $\beta_{-}\left(1+\beta_{-}\right) \gamma_{=}^{2} c / \lambda_{w}$ calculated from the value of $B_{r}$, at constant $\gamma=3.4$ and $B_{*}=13.1 \mathrm{kG}$, that maximizes emission at each frequency. The calculated frequencies of the $\mathrm{TE}_{11}$ and $\mathrm{TM}_{01}$ FEL couplings, and of the $\mathrm{TE}_{11}$ cyclotron maser coupling, are shown, based on the frequencies of crossing intersections.

varying the axial electron velocity through changes in the magnitude of the wiggler magnetic field at constant axial field and constant beam energy, as illustrated in Fig. 5. The theoretical FEL curves in this figure are calculated for the position of the crossing intersections for the dispersion relations of the pump-shifted beam space-charge mode with that of the electromagnetic waveguide modes; note that this is only an estimate of the frequency of maximum growth for the FEL instability. The predicted position of the $\mathrm{TE}_{11}$ cyclotron maser coupling is also illustrated, with its exact location based on a recent paper by H. P. Freund and A. K. Ganguly that predicts a shift in

'Note that this figure differs slightly from that presented in [36].
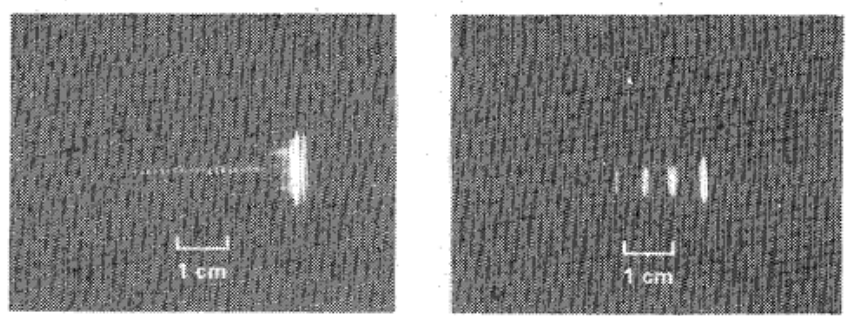

Fig. 6. Atmospheric-pressure air breakdown standing wave patterns produced at $\gamma=3.4, B_{z}=16 \mathrm{kG}$, and (left) $B_{r}=1.16 \mathrm{kG}$ and (right) $B_{r}=$ $2.8 \mathrm{kG}$, demonstrating tuning of the mission spectrum from $\sim 100 \mathrm{GHz}$ (left) to $\sim 25 \mathrm{GHz}$ (right).

the effective cyclotron frequency due to the presence of the wiggler magnetic field [41]. (This paper also generalizes the three-dimensional orbit theory somewhat by considering perturbations about axicentered orbits as well as by analyzing the case of non-axis-encircling trajectories.) The experimental points in this figure are in reasonable agreement with predictions for an FEL interaction; however, this case corresponds to operation somewhat closer to gyroresonance than that of Fig. 3, so that the frequency distinction between the FEL and cyclotron maser is smaller. Broad instantaneous growth bandwidths at $\gtrsim 1.5$ $\mathrm{dB} / \mathrm{cm}$ were experimentally measured, over the range of $65-90 \mathrm{GHz}$, by varying the length of the interaction region, thus demonstrating the important potential of the millimeter-wave FEL as a broad-band amplifier. These broad bandwidths are consistent with the predicted operating characteristics for an FEL operating above gyroresonance with $\Phi<0$ [19]. The production of atmospheric-pressure air plasmas further confirmed the high millimeter-wave powers produced, demonstrated the coupling to both axicentered and hollow modes, as suggested by the grating spectrometer measurements, and allowed a simple demonstration of frequency tuning.

Tuning of the emission at high power over the range 25$100 \mathrm{GHz}$ by varying the magnitude of the wiggler magnetic field at constant $B_{z}$ and $\gamma$ is demonstrated in Fig. 6, which shows two air breakdown standing wave patterns, produced in full atmospheric pressure laboratory air by focusing the millimeter-wave FEL radiation in the vicinity of a metal plate using a $9.3 \mathrm{~cm}$ focal length Teflon lens. Breakdown occurs at half-wavelength intervals beginning at a quarter wavelength from the metal surface, allowing a simple experimental determination of the principal frequency component of the radiation. Microwave-induced breakdown of atmospheric-pressure air requires incident irradiances in the range of $3-5 \mathrm{MW} / \mathrm{cm}^{2}$ for a $15 \mathrm{~ns} \mathrm{ra}-$ diation pulse, but the required power is reduced by a factor of 4 at the antinodes of the standing-wave electric field. Tuning with wiggler field clearly affects the gain and possible saturation of the experiment, and not just the emission frequency. The case of $B_{r}=1.16 \mathrm{kG}$ corresponds to $\beta_{\perp} \sim 0.23$, while the case of $B_{r}=2.8 \mathrm{kG}$ corresponds to $\beta_{\perp} \sim 0.43$. These two cases correspond approximately to the two extremes of the wiggler tuning range at constant $\gamma=3.4$ and $B_{z}=16 \mathrm{kG}$ that still produce sufficient power to produce an atmospheric pressure air plasma. In fact, in 
the second case substantial beam scraping will occur due to the ampltiude of the wiggler-induced helical motion. The observed frequencies are in reasonable agreement with the predicted coupling frequencies shown in Fig. 4 for these values of $\beta_{\perp}$.

However, superradiant FEL experiments have several important drawbacks. In such experiments, initial conditions, radiation growth, and saturation are intimately linked by the choice of experimental parameters, and they are thus difficult to separately characterize and to optimize. The broad emission linewidths that occur in such experiments also complicate theoretical modeling. In addition, superradiant FEL devices are likely to have limited practical application because of their lack of coherence (broad emission linewidths).

\section{Amplifier Experiments}

For these reasons, a true traveling-wave FEL amplifier experiment was assembled in 1983 [42]. The amplifier is a more practical FEL configuration and, furthermore, permits more straightforward single-frequency gain and saturation measurements with which to perform quantitative comparisons with the predictions of theory. This amplifier experiment was the first high-power, high-gain FEL amplifier driven by an intense relativistic electron beam, as well as the first true long-wavelength FEL amplifier of any kind since the early ubitron experiments of R. M. Phillips [2], [3].

In order to make use of an available high-power magnetron source, the amplifier experiment was designed to operate at $35 \mathrm{GHz}$. A directional sidewall input coupler was fabricated to permit signal injection in a vertically polarized $\mathrm{TE}_{11}$ mode without perturbing the electron beam. This was accomplished using two resonant sidewall slots separated by $\frac{3}{4}$ of a wavelength, and driven $90^{\circ}$ out of phase by a short-slot hybrid coupler. The level of the injected signal could be varied using a high-power attenuator consisting of a phase-shifter and two hybrid waveguide tees. In addition, the experimental parameters were adjusted to optimize performance at this frequency. To provide a better match to the lower frequency operation, the voltage of the VEBA accelerator was lowered to produce a beam of $600 \mathrm{~A}$ at $900 \mathrm{keV}$. The wiggler and axial field strengths were selected to produce a $\beta_{\perp}$ of order 0.3 at a reasonable separation from gyroresonance, and then experimentally optimized for gain and saturated emission at $35 \mathrm{GHz}$.

The remaining experimental parameters were $B_{z}=$ $11.75 \mathrm{kG}$ and $B_{r}=1.15 \mathrm{kG}$, yielding $\beta_{\perp}=0.32$ and $\beta_{z}=$ 0.87 . The uncoupled dispersion curves for the FEL amplifier are shown in Fig. 7, which shows the pump-shifted beam space-charge line, $\omega=\left(k+k_{w}\right) v_{z}$, for $\beta_{z}=$ $0.87 \pm 0.01$, crossing the line $\omega^{2}=k^{2} c^{2}+\omega_{\mathrm{co}}^{2}$, where $\omega_{\mathrm{co}}$ is the cutoff for the $\mathrm{TE}_{11}$ waveguide mode in the $10.8 \mathrm{~mm}$ i.d. cylindrical interaction waveguide. This figure depicts a near-tangent intersection of these two curves in the vicinity of $35 \mathrm{GHz}$. For reference, the position of the Dop-

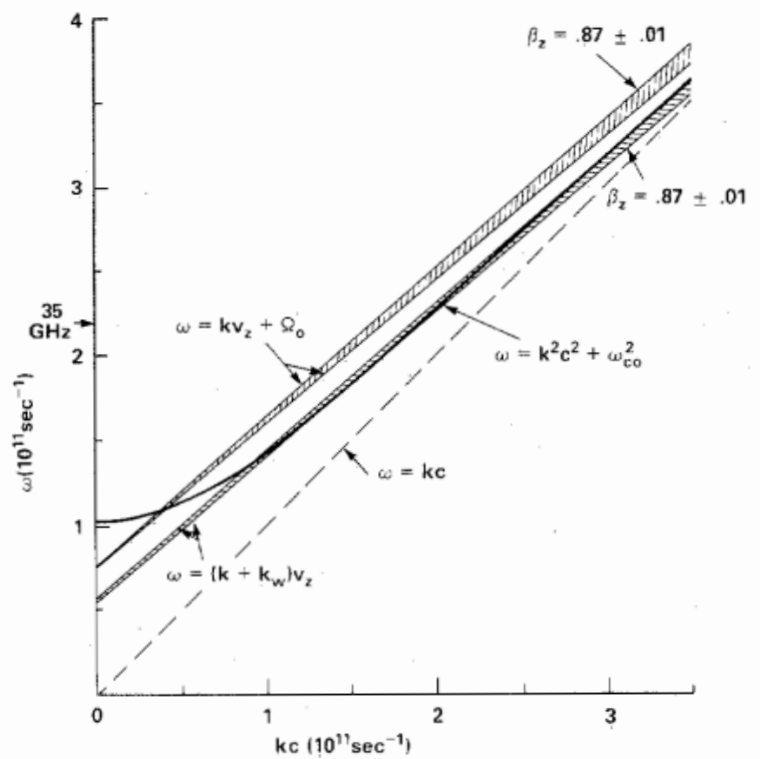

Fig. 7. Uncoupled dispersion curves for the $35 \mathrm{GHz}$ FEL amplifier.

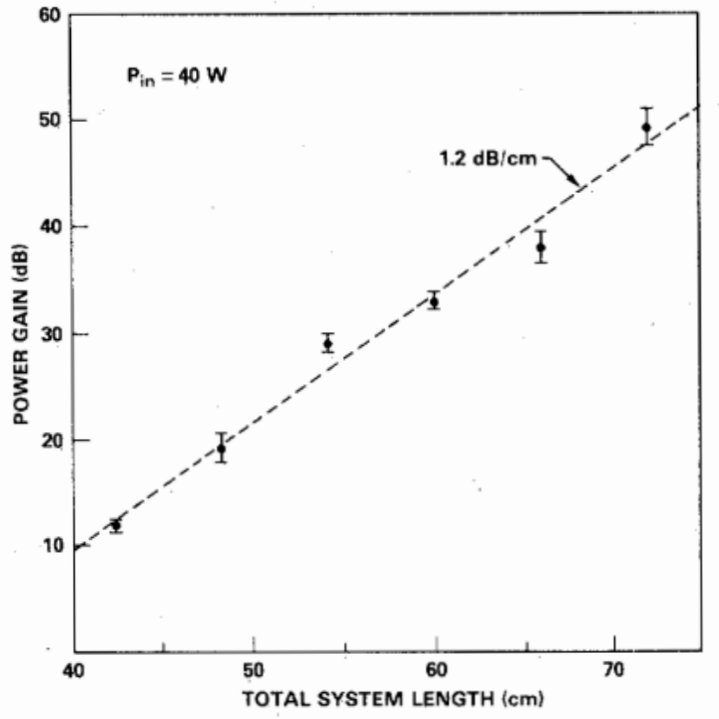

Fig. 8. Power gain versus system length for the $35 \mathrm{GHz}$ FEL amplifier.

pler-shifted cyclotron maser line, $\omega=k v_{z}+\Omega_{0}$, is also shown for the same values of $\beta_{z}$. For these parameters, this line is well separated from the space-charge line.

Fig. 8 illustrates the linear growth of a $40 \mathrm{~W}$ signal as a function of interaction length, which was varied by changing the length of the axial field magnet. (Total system length in the figure is arbitrarily defined to include fixed length regions of wiggler entry taper and axial field exit taper that do not contribute to the linear gain.) The use of a small injected signal prevented saturation of the amplified signal at total gains of order $50 \mathrm{~dB}$. Here power gain is defined as the ratio of vertically polarized output power to vertically polarized input power. Since the gain is expected to be circularly polarized (and the emission is found experimentally to be equal in both linear polarizations), the true gain is believed to be $6 \mathrm{~dB}$ larger than illustrated.

Operating at $35 \mathrm{GHz}$ with these experimental parame- 
ters as a true traveling-wave amplifier, the FEL has demonstrated high linear gain $(>50 \mathrm{~dB})$ at a high spatial growth rate $(1.2 \mathrm{~dB} / \mathrm{cm})$, high power $(17 \mathrm{MW})$ at good efficiency ( $>3$ percent), and narrow emission linewidth $(<300 \mathrm{MHz})$. These results are a confirmation of the potential of FEL's as high-gain amplifiers and as high-power coherent sources of millimeter waves, as well as a demonstration of a unique set of experimental capabilities. These capabilities include the ability to produce powers in excess of $10 \mathrm{MW}$ at $35 \mathrm{GHz}$ in the fundamental $\mathrm{TE}_{\mid 1}^{o}$ mode with input powers of less than $100 \mathrm{~W}$, and the ability to generate very short (nanosecond) pulses at these power levels.

\section{Linear Induction Accelerator Experiment}

\section{A. Background}

Free-electron laser experiments using the linear induction accelerator (LIA) began in 1981. The experiments were motivated by the uniquely long pulse duration $(2 \mu \mathrm{s})$ of this accelerator. The pulse duration of other high-power millimeter wavelength FEL experiments has typically been on the order of a round-trip transit time of the radiation through the wiggler. In contrast, approximately 200 round trips of the radiation can take place during the $2 \mu$ s pulse of this experiment, so that a study of steady-state behavior can be performed which is directly applicable to various systems requiring $\mathrm{CW}$ or quasi-CW sources. Also, a long pulse will be required to operate in the oscillator mode, and it simplifies the study of saturation and other nonlinear effects.

The first LIA-FEL experiments used an axial field of $1-5 \mathrm{kG}$ to confine the beam as it propagated through the wiggler [43], [44]. Three different types of wiggler (diffusive, planar, and helical) were used, and the radiation generated with each of them was studied in some detail. In particular, cyclotron emission was always observed whenever an axial magnetic field was present. The emission spectra typically contained many peaks, corresponding to various waveguide modes and at least two cyclotron harmonics, in addition to the desired FEL radiation. Consequently, the results were often ambiguous, and particular peaks could be positively identified only with great difficulty.

The cyclotron emission was particularly difficult to eliminate because the LIA electron gun is located in a null magnetic field. Therefore, when electrons were injected into the axial field used to guide them through the FEL interaction region, they acquired a significant azimuthal velocity (as required to maintain zero canonical angular momentum). This beam rotation greatly facilitated the cyclotron emission. Another problem encountered with the planar wiggler in combination with the guide field was a gradient drift which tended to cause the beam to be lost to the drift tube wall [45].

To avoid these problems, the axial magnetic field was eliminated and the radial focusing property of the helical wiggler was used to confine the electron beam. The elim- ination of the axial field simplified both the experiment and the interpretation of the results. Another advantage of eliminating the axial field is that scaling to higher radiation frequencies is more straightforward. An FEL operating near the cyclotron resonance would require a prohibitively large axial magnetic field at wavelengths much shorter than $1 \mathrm{~mm}$.

In this section, we will only describe experiments in which all the beam focusing and guiding is provided by a helical wiggler having a field amplitude on axis of $B_{r} \approx$ $1 \mathrm{kG}$. Although the beam current transported through the wiggler is about half that of previous FEL experiments using this accelerator [43], [44] with $B_{z} \sim 2-4 \mathrm{kG}$, the FEL output power is about an order of magnitude higher and the spectrum is much clearer. Up to $4 \mathrm{MW}$ of $\sim 30$ $\mathrm{GHz}$ radiation has been generated from a $650 \mathrm{keV}, 200 \mathrm{~A}$ beam [46].

\section{B. Description of Long-Pulse FEL Experiment}

The electron beam which drives the long-pulse FEL is generated by a linear induction accelerator (LIA), a schematic of which is shown in Fig. 9. The accelerator was designed and constructed at the National Bureau of Standards [47], and its unique feature is a $2 \mu$ s duration pulse. The injector, which is normally operated at $350-450 \mathrm{kV}$, consists of a pulse-forming network and a 12:1 step-up transformer powering an Astron II electron gun. This gun has an $11 \mathrm{~cm}$ anode-cathode spacing, and the cathode is located in an essentially null magnetic field $\left(B_{z} \leqslant 3 \mathrm{G}\right)$. A series of solenoidal coils is used to focus and transport the beam through the rest of the accelerator. The induction modules consist of two iron core units pulsed by separate modulators. Each of the units inductively drives an accelerating gap, which is typically run at $100-150 \mathrm{kV}$ in these experiments. Consequently, the final beam energy is variable between 550 and $750 \mathrm{keV}$.

Although the electron gun was originally designed to operate with a thermionic cathode, the experiments described here have all been performed with a graphite brush cathode [48]. This $15 \mathrm{~cm}$ diameter cathode is fabricated by weaving graphite fiber yarn (Thornel, by Union Carbide) through an aluminum plate. The approximately $1 \mathrm{~mm}$ thick threads of $\sim 10 \mu \mathrm{m}$ fibers are spaced $1 \mathrm{~cm}$ apart and are trimmed so that they extend $1 \mathrm{~cm}$ from the surface of the support plate. The fibers enhance the electric field, which averages only $\sim 40 \mathrm{kV} / \mathrm{cm}$ without enhancement, so that electrons are field emitted at room temperature. This cathode has proven to be very reliable, long-lived (thousands of pulses with no evidence of deterioration), and has greatly simplified the operation of the accelerator. The gun operates satisfactorily at pressures of up to $10^{-5}$ Torr, but beyond that point the accelerating gap insulators routinely flash over.

The two obvious drawbacks to this type of cathode for the present application are the increased beam emittance (due to the highly nonuniform emitting surface) and the tendency for the cathode plasma which is produced during 


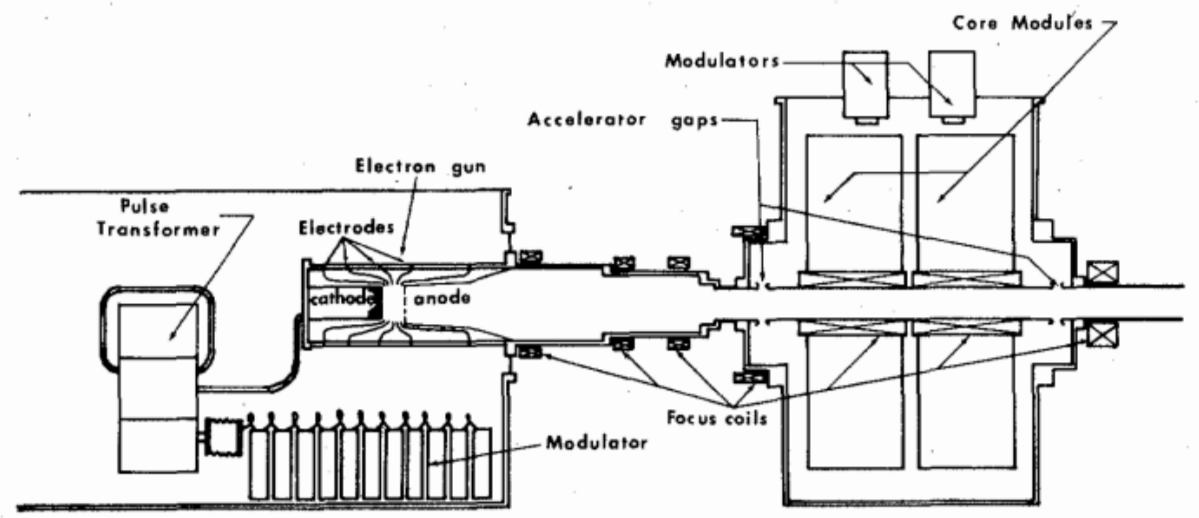

Fig. 9. Schematic diagram of linẹar induction accelerator.

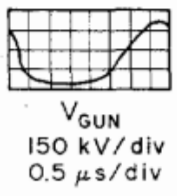

Fig. 10. LIA electron gun voltage and current waveforms with graphite brush cathode.

the field emission to expand toward the anode, thereby reducing the gun impedance during the $2 \mu$ s pulse. From the injector voltage and current waveforms shown in Fig. 10, we see that both the voltage and the current remain quite constant during most of the pulse, but that the voltage begins to drop substantially at $\sim 1.5 \mu$ s into the pulse. Until that time, the voltage drop is approximately 5 percent, which is more than the 2 percent observed with a thermionic cathode but substantially less than the $20-30$ percent one would expect for plasma velocities of $2-2.5 \mathrm{~cm} /$ $\mu \mathrm{s}$, as have been observed with higher current density cathodes.

The beam emittance is measured by passing the beam through a mask containing $3 \mathrm{~mm}$ diameter holes and measuring the expansion of the resulting beamlets as they strike a target $2-10 \mathrm{~cm}$ away. In these measurements, only the first focusing coil is used, and the mask is placed 70 $\mathrm{cm}$ downstream of the coil where the beam current is $800 \mathrm{~A}$ and $B_{z}<5 \mathrm{G}$. The target is a $0.2 \mathrm{~mm}$ thick molybdenum sheet backed with $1 \mathrm{~mm}$ thick NE 102 scintillator. For time-integrated measurements, an open shutter camera photographs the entire $20 \mathrm{~cm}$ diameter beam cross section. Time-resolved measurements are performed by taking streak photographs of one or two individual beamlets. Microdensitometer scans of the resulting photographs are shown in Fig. 11. From these measurements, we find that the maximum angular beamlet divergence is $r^{\prime}=\Delta r / \Delta z \approx 0.014$ and that this divergence is quite constant during the $2 \mu$ s pulse. Consequently, the normalized beam emittance is found to be $\epsilon_{n}=\beta \gamma \pi r r^{\prime} \approx 0.25 \pi$ $\mathrm{rad} \cdot \mathrm{cm}$. This value is somewhat larger than the $0.14 \pi$ $\mathrm{rad} \cdot \mathrm{cm}$ which was measured at NBS with a thermionic cathode and slightly lower beam current. To optimize the transport of this higher emittance beam, the accelerator transport field had to be increased by about a factor of 2

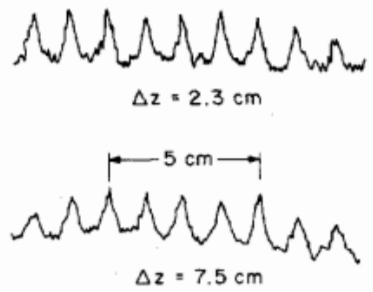

(a)

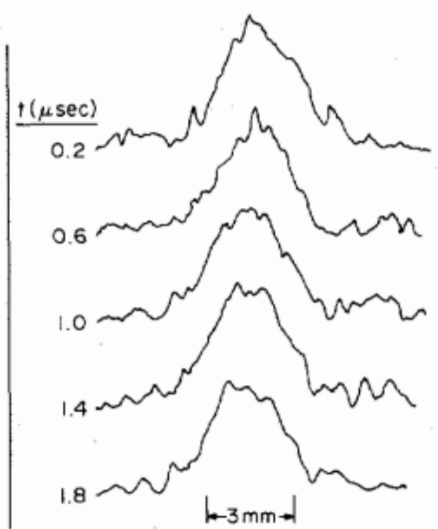

(b)
Fig. 11. Emittance measurements of LIA beam. (a) Time integrated measurements at two target-mask separations $\Delta z$. (b) Time resolved measurement of a single beamlet with $\Delta z=7.5 \mathrm{~cm}$.

over the NBS value. With this modified transport field, a beam current of $600 \mathrm{~A}$ is extracted through a $2.5 \mathrm{~cm}$ diameter aperture at the end of the accelerator.

The wiggler is a bifilar helix having a $4 \mathrm{~cm}$ period and $4 \mathrm{~cm}$ diameter. The windings consist of two layers of 1.0 $\mathrm{cm}$ wide $\times 1.25 \mathrm{~mm}$ thick copper wire. The total wiggler length is $128 \mathrm{~cm}$ (32 periods), including a six-period adiabatic transition at each end. At the end of a bifilar helix, where the winding reverses direction, there is a current loop which generates a large field perturbation [49]. We have reduced this perturbation to $<3$ percent of the peak field by placing an external compensating winding at the entrance end of the wiggler. The $3 \mathrm{~cm}$ i.d. $\times 0.75 \mathrm{~mm}$ thick stainless steel beam tube passing through the wiggler allows the pulsed wiggler field (100 $\mu$ s rise time) to penetrate nearly unperturbed to the axis. A $1 \mathrm{kG}$ field on axis is generated by pulsing a current of $8 \mathrm{kA}$ through the windings.

One of the major advantages of the helical wiggler is that it provides radial focusing for the beam. Thus, it is possible to propagate a relatively large beam current through the wiggler with no external focusing. Of course, the expansion forces on the beam (emittance and space charge) must be balanced by the focusing force for confinement, so there is a limit to the beam current that can 


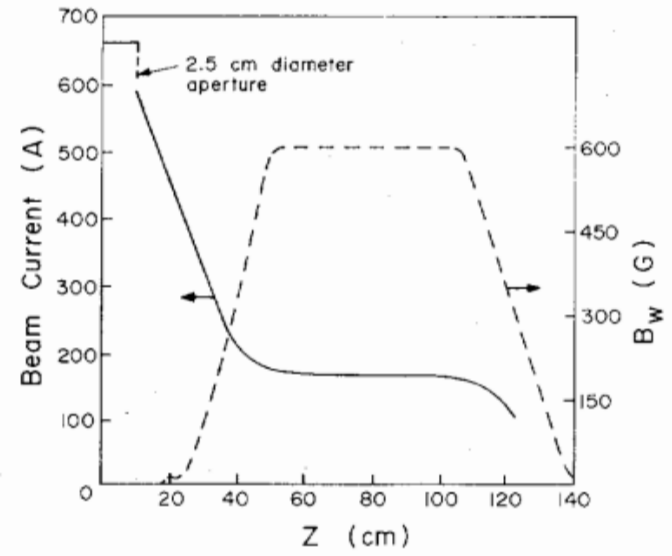

Fig. 12. Beam current (solid line) and wiggler field amplitude (dashed line) versus axial distance from end of LIA.

be propagated. A plot of current transport through the wiggler is shown in Fig. 12. As the beam expands from its focus at the entrance to the wiggler, the current decreases until the beam reaches the constant-amplitude portion of the wiggler. The current decrease in this transition region is a function of $B_{r}$, but if $B_{r} \geq 300 \mathrm{G}$ there is no discernible additional current loss through the constantamplitude part of the wiggler. At the end of the wiggler, the beam is diverted to the wall of the beam tube, either by free expansion or with a perpendicular magnetic field.

Measurements of the beam position and size in the wiggler are performed using a sliding molybdenum target with an attached scintillator which is photographed from the end of the wiggler. The beam radius is typically $\sim 0.5 \mathrm{~cm}$, but the beam centroid is off axis by as much as $0.5 \mathrm{~cm}$. Consequently, the beam executes a spiral motion as it propagates through the wiggler. The off-centering of the beam is indicative of the difficulty of properly matching the beam into the wiggler. This problem is much greater in this experiment, where the beam has to be propagated over a distance of about $3 \mathrm{~m}$ from the gun to the FEL, than in devices with the wiggler located immediately downstream from the beam diode.

The radiation is normally coupled out of the device through a conical horn and a $15 \mathrm{~cm}$ diameter Teflon vacuum window. Various diagnostics are then used to measure the power and frequency of the radiation.

\section{Experimental Results}

Because the wiggler provides all the electron beam focusing in this experiment, one might expect a strong dependence of the FEL performance on the wiggler field amplitude. The variation of radiated power and beam current with wiggler field is shown in Fig. 13. Although the propagated current increases almost linearly with wiggler field up to about $750 \mathrm{G}$, there is a sharp threshold at $B_{r} \approx 400 \mathrm{G}$ for the generation of significant FEL radiation. Both the transported current and the microwave power peak at $B_{r} \sim 625-750 \mathrm{G}$. The ratio of radiated power to beam current is nearly constant (to within the \pm 10 percent experimental reproducibility) from $B_{r}=500$ $\mathrm{G}$ to $1 \mathrm{kG}$.

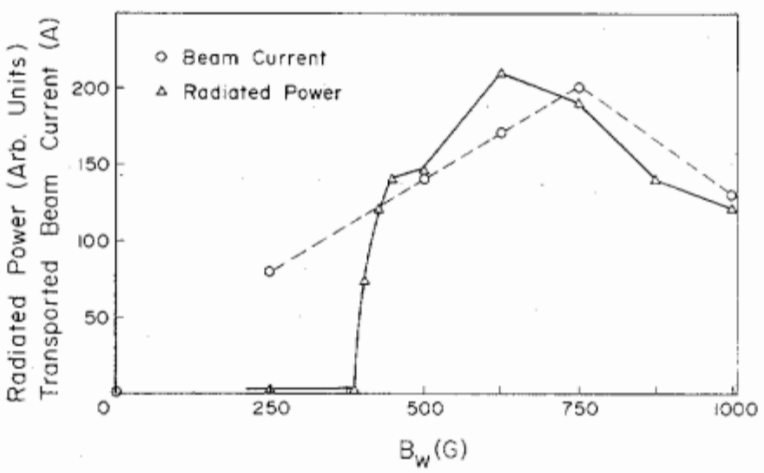

Fig. 13. Variation of propagated beam current and radiated power with wiggler field amplitude.

As in the pulseline accelerator experiments discussed in Section II, theoretical output frequencies are given by the intersections of the waveguide mode dispersion curve and the FEL-beam line: $\omega=\left(k+k_{w}\right) v_{z}$. For $\omega_{b}<\omega_{c o}$, where $\omega_{b}$ is the beam plasma frequency and $\omega_{\text {co }}$ is the cutoff frequency of a particular waveguide mode, it is easy to show that

$$
\omega=k_{w} v_{z} \gamma_{z}^{2}\left[1 \pm \beta_{z}\left(1-\omega_{\mathrm{co}}^{2} / k_{w}^{2} v_{z}^{2} \gamma_{z}^{2}\right)^{1 / 2}\right]
$$

Normally, in this experiment, output is observed at both the high and low frequency intersections, but the low-frequency output is typically about an order of magnitude lower in power. In normal operation, the beam energy is $E_{b}=650-700 \mathrm{keV}, B_{r}$ is $\leq 1 \mathrm{kG}$, and the high frequency output peaks are at $30-35 \mathrm{GHz}$.

The frequency of the output radiation has been determined by using a series of cutoff filters, a gas-breakdown spectrometer [50], and a Fabry-Perot interferometer [51]. All three diagnostics give the same center frequency, but the Fabry-Perot interferometer gives more precise spectral width information. This interferometer consists of two $3 \mathrm{~mm}$ thick polyethylene sheets which have conducting paint sprayed on one side to form partially transmitting mirrors. Radiation from a $K_{a}$-band transmitting horn impinges on the fixed mirror, and a receiving horn collects radiation passing through the movable mirror. Standard crystal detectors monitor the input and output of the interferometer. Two sets of Fabry-Perot data are shown in Fig. 14. Each point represents a single shot, and the dashed lines show the calibration curves at the two frequencies. As expected, the output frequency increases with beam energy. By comparing the width of the data peaks to that of the calibration peaks, we find that the linewidth is less than 10 percent. The fact that the FabryPerot output does not drop to as low a value as the calibration curves between peaks is consistent with the presence of an additional mode. Indeed, the high-pass filter data indicate the presence of an additional broad-band component with frequencies from approximately 40 to 60 $\mathrm{GHz}$. However, such a broad-band output cannot be resolved by the interferometer. As will be discussed below, this higher frequency radiation is consistent with the excitation of a second FEL waveguide mode. 


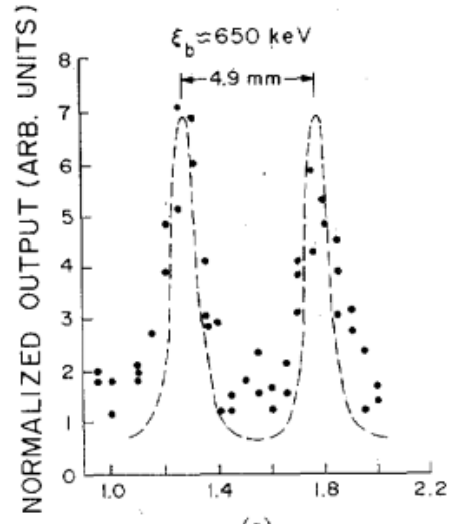

(a)

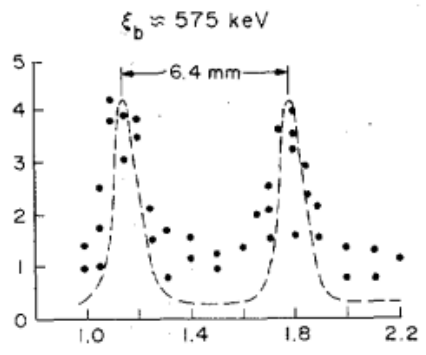

(b)
OUTPUT MIRROR POSITION $(\mathrm{cm})$

Fig. 14. Output of Fabry-Perot interferometer for two different electron beam energies. Each point represents a single shot, and the dashed lines result from a single-frequency calibration.

The high frequency output power is measured with the gas-breakdown spectrometer and with a calorimeter. Again, the results agree to within the accuracy of the diagnostics. The spectrometer consists of a Lucite tube with a lens at one end and a plane copper mirror at the other. Radiation injected through the lens is collimated and reflected from the mirror, thereby creating a standing wave. The gas pressure in the tube is adjusted so that the gas breaks down just at the peaks of the standing wave. The breakdown is photographed and the wavelength and electric field amplitude are determined from the spacing of the breakdown spots and the gas pressure, respectively. Then the power is calculated from the electric field and the size of the spots. The calorimeter [29] consists of a pyramidshaped collector formed from $0.5 \mathrm{~mm}$ thick SynthaneTaylor L-564 absorber. Thermistors mounted on each face of the collector are connected in series to determine the temperature change. The calorimeter has a sensitivity of $0.16^{\circ} \mathrm{C} / \mathrm{J}$. Radiation is injected into the calorimeter through a $1.25 \mathrm{~cm}$ diameter tube, which cuts off the lowfrequency output. Radiation which leaks out the back of the calorimeter is monitored with a crystal detector to determine the pulse shape so that the power can be calculated.

The maximum radiated power is obtained with $E_{b}=$ $700 \mathrm{keV}, I_{b}=200 \mathrm{~A}$, and $B_{r}=625 \mathrm{G}$. A peak power of $4 \mathrm{MW}$ has been measured in this case, as calculated from a measured pulse energy of $1.4 \mathrm{~J}$ and a radiation pulse duration (FWHM) of $0.35 \mu \mathrm{s}$. The relevant oscilloscope traces are shown in Fig. 15(a). Note that the beam current-pulse duration is only about half that of the gun pulse. At the time this experiment was performed, adjusting the transport coils for maximum beam current resulted in the excitation of a cavity instability in the accelerator beam tube. This instability is indicated by the large RF oscillations $(550 \mathrm{MHz})$ shown on the accelerating gap voltage traces. This instability disrupted the beam and shortened the beam and radiation pulse durations. By detuning the transport coils or by aperturing the beam at the gun, we were able to prevent the instability and obtain full $2 \mu \mathrm{s}$ (a)
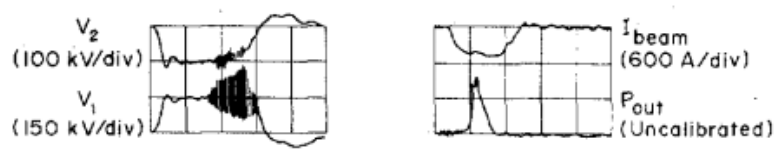

(1 $\mu$ sec/div)
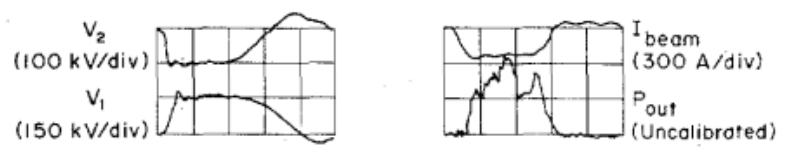

Fig. 15. Oscillographs of the LIA accelerating gap voltages, beam current out of the LIA, and FEL radiation output. (a) LIA tuned for maximum beam current. (b) $7.5 \mathrm{~cm}$ diameter aperture placed just downstream of gun anode.
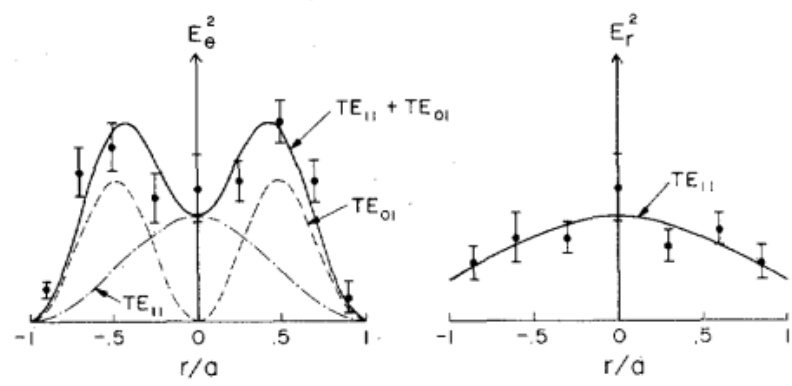

Fig. 16. Azimuthal and radial electric field components of the radiation emerging from the FEL output horn together with calculated profiles of the $\mathrm{TE}_{11}$ and $\mathrm{TE} \mathrm{E}_{01}$ modes.

duration output pulses, as shown in Fig. 15(b). However, because the beam current was only $\$ 100 \mathrm{~A}$ in this case, the peak microwave power was reduced. Subsequently, the beam instability was studied in detail, and was found to originate in the large-diameter section of the beam tube connected to the gun (see Fig. 9). This section of tube forms a cavity having a $\mathrm{TE}_{111}$ mode resonance at exactly the $550 \mathrm{MHz}$ frequency observed on the beam. We were then able to eliminate the cavity oscillation without reducing the beam current by placing a dielectric strip along the wall of the beam tube, or by shorting the $T E_{111} R F$ field in the tube with a thin wire.

To determine the radiation mode structure, a $K_{a}$-band pickup waveguide is placed at various positions on the face of the output window. The waveguide is rotated to detect either $E_{r}$ or $E_{\theta}$, the electric field components of the RF field. The results of these measurement are shown in Fig. 16. Both field components are quite symmetric about the axis, and the results are the same along both the vertical and horizontal diameters. No single mode exists with field components that have the measured profiles, so at least two separate modes must be present. As shown in the figure, a reasonably good fit to the data is obtained by superimposing $\mathrm{TE}_{11}$ and $\mathrm{TE}_{01}$ modes of similar peak amplitudes. Since only the $\mathrm{TE}_{11}$ mode has a radial component, the radial data is used to determine the amplitude of that mode. Then it is found that a $\mathrm{TE}_{01}$ component with a 36 percent higher amplitude is required to give a reasonably good fit to the azimuthal field data. Also, the observed symmetry is consistent with the generation of a circularly polarized $\mathrm{TE}_{11}$ mode, as expected with the helical wiggler. 


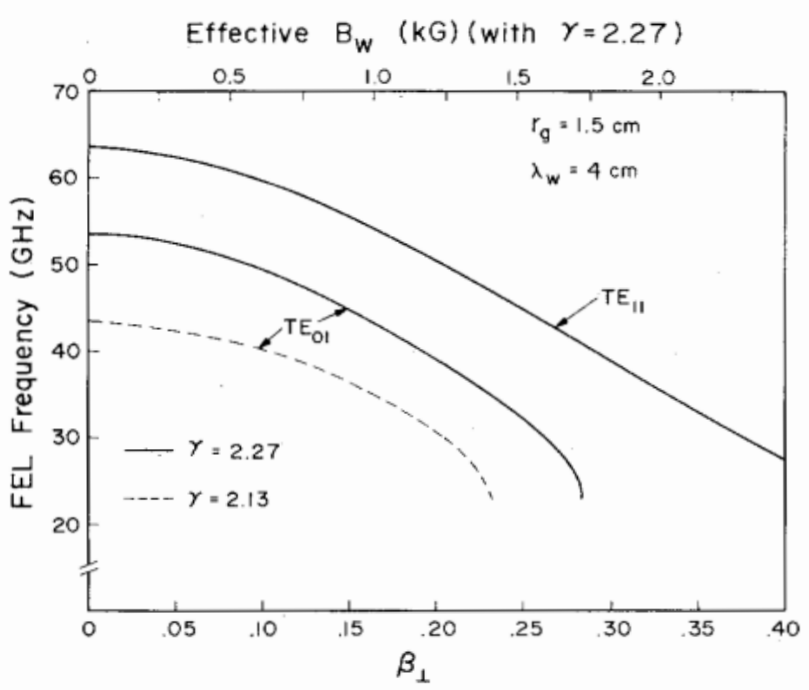

Fig. 17. Theoretical FEL interaction frequencies for the two waveguide modes as a function of perpendicular electron velocity. Also shown is the wiggler field amplitude required to generate that $\beta_{\perp}$, assuming no other contribution.

\section{Discussion}

Both the Fabry-Perot interferometer and the high-pass filters show the predominant frequency to be $30-33 \mathrm{GHz}$ when $B_{r}=1 \mathrm{kG}$ and $E_{b}=650 \mathrm{keV}$. This frequency is somewhat less than would at first be expected from (1) using a $\gamma_{z}$ determined from both the total $\gamma$ and the perpendicular velocity due to the wiggler: $\beta_{\perp}=\Omega_{r} / c k_{w}$, where $\Omega_{r}=e B_{r} / \gamma m c$. For the above parameters, $\beta_{\perp}=0.16$. Fig. 17 shows the predicted output frequencies for the $\mathrm{TE}_{01}$ modes as a function of $\beta_{\perp}$ and as a function of the wiggler field required to produce that $\beta_{\perp}$. Note that an effective wiggler field of $1.5-1.6 \mathrm{kG}$ or $2.1-2.3 \mathrm{kG}$ would be required to generate $30 \mathrm{GHz}$ radiation in the $\mathrm{TE}_{01}$ or $\mathrm{TE}_{11}$ mode, respectively, assuming that the perpendicular velocity is due only to the wiggler. To a large degree, this apparent discrepancy can be explained by the experimental observation that the beam centroid is $\sim 0.5 \mathrm{~cm}$ off axis. The off-axis electrons experience a higher wiggler field and undergo betatron oscillations, both of which increase $\beta_{\perp}$. The $\mathrm{TE}_{01}$ field intensity peaks at $r / a=0.5(r=0.75$ $\mathrm{cm}$ ), at which point the wiggler field is calculated to be 58 percent larger than the field on axis. It is not unreasonable to expect that the outer electrons couple most strongly to the $\mathrm{TE}_{01}$ mode. These electrons are in a wiggler field that is large enough to account for the observed frequency in that mode.

The higher frequency broad-band emission that is observed using the high-pass filters is presumably the $\mathrm{TE}_{11}$ emission that is detected during the mode measurements. Although this emission has not been studied as carefully as the narrower band emission at $\sim 30 \mathrm{GHz}$, the observed frequency range of $40-60 \mathrm{GHz}$ is roughly consistent with the expected output frequencies given the range of wiggler fields sampled by the beam. A quantitative, three-dimensional analysis of the interaction, which would be required to calculate the theoretical coupling, is very difficult and beyond the scope of the present work. However, it is in- teresting to note that the narrower band output is apparently in the $\mathrm{TE}_{01}$ mode, which has a relatively narrow intensity profile across the waveguide. Also, as shown in Fig. 17, electrons with $\beta_{\perp}>0.28$ cannot interact with the mode. Thus, it is plausible that only electrons in a relatively small annular region interact strongly with the $\mathrm{TE}_{01}$ mode. These electrons could be expected to have a smaller axial velocity spread, at least due to the wiggler field gradient, than the full beam. Thus, the $\mathrm{TE}_{01}$ output might be expected to have a narrower linewidth than the $\mathrm{TE}_{11}$ mode, which has a broad profile that encompasses the full beam.

Assuming then that the narrow-band output is in the $\mathrm{TE}_{01}$ mode, we can analyze the observed tuning of the output with total beam energy. When $E_{h}$ is decreased to $\sim 575 \mathrm{keV}(\gamma \approx 2.13)$, the output frequency drops to $\sim 24$ $\mathrm{GHz}$. Theoretically, this emission should be generated by electrons with $\beta_{1}=0.23$, as shown in Fig. 17. Assuming that the electrons continue to "see" an effective wiggler field of $1.5 \mathrm{kG}$ in this case, then $\beta_{\perp}$ would be 0.26 . However, electrons with $\beta_{\perp}=0.26$ do not resonate with the $\mathrm{TE}_{01}$ mode (because the FEL-beam line does not intersect the $\mathrm{TE}_{01}$ dispersion curve). As shown in the figure, the largest $\beta_{\perp}$ for which resonance can occur is $\beta_{\perp}=0.23$, in which case the FEL-beam line intersects the $\mathrm{TE}_{01}$ dispersion curve tangentially. Consequently, the observed frequency shift is as large as can be achieved in the $\mathrm{TE}_{01}$ mode, even though the resonant electrons have a slightly lower $\beta_{\perp}$ than we would predict by simply scaling the higher beam energy results.

The peak power of $4 \mathrm{MW}$ corresponds to an interaction efficiency of 3 percent. The theoretical cold-beam efficiency [8], $\eta=\omega_{b} /\left(\gamma^{1 / 2} \gamma_{z} c k_{w}\right)$, where $\omega_{b}$ is the beam plasma frequency, is $\eta \approx 10$ percent. Of course, it is not clear that saturation has been reached in this single-pass, superradiant experiment; but given the beam energy spread and the off-axis propagation of the beam through the wiggler, the experimental efficiency is not expected to be as large as the 1-D, cold-beam theoretical value.

A numerical analysis of this experiment using the 1-D nonlinear theory, described in [52], has been performed by C, M. Tang. She finds that the theoretical efficiency can be increased from $\sim 5$ percent with a constant parameter wiggler to $>25$ percent with a wiggler having an appropriately tapered amplitude and/or period. A representative result is shown in Fig. 18. An experimental study of efficiency enhancement using tapered wigglers is planned.

Work is in progress to improve the beam quality and the beam centering in the wiggler. This should enhance the excitation of the fundamental $\mathrm{TE}_{11}$ mode. The success of the experiment to date suggests that a repetitively pulsed, long-pulse, high-power FEL would be an attractive means of delivering high energy millimeter-wavelength radiation. The long-pulse capability of this experiment makes an oscillator configuration very attractive. Furthermore, the long pulse duration together with the high current level make a high-power two-stage oscillator feasible [53], [54]. A two-stage FEL driven by the present electron beam is 


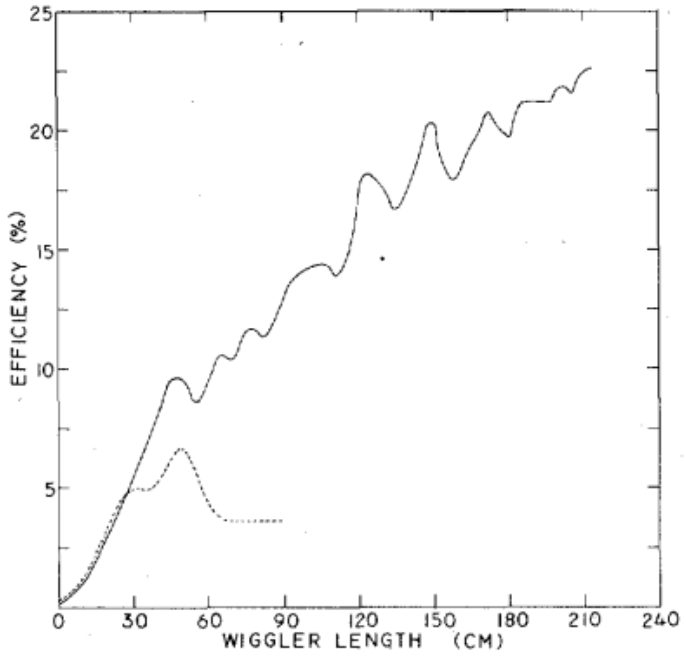

Fig. 18. Theoretical interaction efficiency versus wiggler length with a tapered and an untapered wiggler. Beam parameters are those of the longpulse induction linac.

capable of operating at submillimeter wavelengths, and with a modest increase in beam energy it should be possible to reach the infrared or even shorter wavelengths. We are currently addressing these issues.

\section{Conclusions}

The history of long-wavelength FEL research traces directly from the early low-voltage ubitron experiments of R. M. Phillips at General Electric in the late 1950's and early 1960 's. Early attempts to transfer this concept to the higher currents and voltage available from pulseline accelerators (kiloamperes at megavolts) produced record powers at a variety of wavelengths, from submillimeter to centimeter waves, but at vanishingly low efficiencies ( 0.003-0.2 percent). Two recent sets of experiments have been carried out at NRL, using a magnetized beam from a pulseline accelerator, and an unmagnetized beam from a long-pulse linear induction accelerator. Each of these experiments has succeeded in demonstrating successful FEL operation, producing megawatts of millimeter-wave radiation with efficiencies in the range of 3-6 percent. The short-pulse FEL based on a pulseline accelerator has succeeded in operating both as a superradiant amplifier in the range of $25-100 \mathrm{GHz}$ and as a true highgain amplifier at $35 \mathrm{GHz}$. Further experiments on it are planned in the near future. The long-pulse FEL based on the induction linear accelerator has also operated successfully as a superradiant amplifier, and will be used for oscillator experiments in the near future. The results of these experiments have aroused strong interest in further investigations of long wavelength FEL devices. A growing list of new long-wavelength FEL experiments at a number of different laboratories, both planned and already under way, will further explore and extend the parameter space that these two experiments have pioneered.

\section{ACKNOWLEDGMENT}

We wish to acknowledge the contributions of our past and present colleagues in the Beam Dynamics Group, the
High Power Electromagnetic Radiation Branch, and the Plasma Theory Branch at NRL, many of whom are cited as coauthors on the various papers describing aspects of this work.

\section{REFERENCES}

[1] M. Friedman and M. Herndon, "Microwave emission produced by the interaction of an intense relativistic electron beam with a spatially modulated magnetic field," Phys. Rev. Lett., vol. 28, pp. 210-212, 1972; "Emission of coherent microwave radiation from a relativistic electron beam propagating in a spatially modulated field," Phys. Fluids, vol. 16, pp. 1982-1995, 1973.

[2] R. M. Phillips, 'The Ubitron, a high-power traveling-wave tube based on a periodic beam interaction in unloaded waveguide," IRE Trans. Electron Devices, vol. ED-7, pp. 231-24I, 1960.

[3] C. E. Enderby and R. M. Phillips, "The Ubittron amplifier-A highpower millimeter-wave TWT," Proc. IEEE, vol. 53, p. 1648, 1965.

[4] P. Sprangle, V. L. Granatstein, and L. Baker, 'Stimulated collective scattering from a magnetized relativistic electron beam," Phys. Rev. $A$, vol. 12, pp. 1697-1701, 1975.

[5] L. R. Elias, W. M. Fairbank, J. M. J. Madey, H. A. Schwettman, and T. I. Smith, "Observation of stimulated emission of radiation by relativistic electrons in a spatially periodic transverse magnetic field," Phys. Rev. Lett., vol. 36, pp. 717-720, 1976.

[6] D. A. G. Deacon, L. R. Elias, J. M. J. Madey, G. J. Ramian, H. A. Schwettman, and T. I. Smith. "First operation of a free-electron laser," Phys. Rev. Lett., vol. 38, pp. 892-894, 1977.

[7] N. A. Kroll and W. A. McMullin, "Stimulated emission from relativistic electrons passing through a spatially periodic transverse magnetic field," Phys. Rev. A, vol. 17, pp. 300-308, 1978.

[8] P. Sprangle, R. A. Smith, and V. L. Granatstein, "Free electron lasers and stimulated scattering from relativistic electron beams," in Infrared and Millimeter Waves, Vol. I: Sources of Radiation, K. J. Button, Ed. New York: Academic, 1979, pp. 279-327.

[9] M. Friedman and M. Herndon, "Generation of intense infrared radiation from an electron beam propagating through a rippled magnetic field," Appl. Phys. Lett., vol. 22, pp. 658-660, 1973.

[10] V. L. Granatstein, M. Herndon, R. K. Parker, and S. P. Schlesinger, "Strong submillimeter radiation from intense relativistic electron beams," IEEE Trans. Microwave Theory Tech., vol. MTT-22, pp. $1000-1005,1974$.

[11] V. L. Granatstein, S. P. Schlesinger, M. Herndon, R. K. Parker, and J. A. Pasour, "Production of megawatt submillimeter pulses by stimulated magneto-Raman scattering," Appl. Phys. Lett., vol. 30, pp. 384-386, 1977.

[12] P. C. Efthimion and S. P. Schlesinger, "Stimulated Raman scattering by an intense relativistic electron beam in a long rippled magnetic field," Phys. Rev. A, vol. 16, pp. 633-639, 1977.

[13] T. C. Marshall, S. Talmadge, and P. Efthimion, "High-power millimeter radiation from an intense relativistic electron beam device," Appl. Phys. Lett., vol. 31, pp. 320-322, 1977.

[14] R. M. Gilgenbach, T. C. Marshall, and S. P. Schlesinger, "Spectral properties of stimulated Raman radiation from an intense relativistic electron beam," Phys. Fluids, vol. 22, pp. 971-977, 1979.

[15] D. B. McDermott, T. C. Marshall, S. P. Schlesinger, R. K. Parker, and V. L. Granatstein, "High-power free-electron laser based on stimulated Raman backscattering," Phys. Rev. Lett., vol. 41, pp. 1368$1371,1978$.

[16] T. J. Orzechowski, M. C. Moebus, F. A. Penko, D. Prosnitz, D. Rogers, C. S. Chavis, K. Halbach, D. B. Hopkins, R. W. Kuenning, A. C. Paul, A. M. Sessler, G. D. Stover, J. T. Tanabe, R. M. Yamamato, and J. S. Wurtele, "The status of the Lawrence Berkeley Laboratory and the Lawrence Livermore National Laboratory (LLNL) free electron laser (FEL)," in Free-Electron Generators of Coherent Radiation, C. A. Brau, S. F. Jacobs, and M. O. Scully, Eds., Proc. SPIE, vol. 453 , pp. $65-74,1984$.

[17] L. Friedland, "Electron beam dynamics in combined guide and pump magnetic fields for free electron laser applications," Phys. Fluids, vol. 23 , pp. 2376-2382, 1980.

[18] P. Diament, "Electron orbits and stability in realizable and unrealizable wigglers of free-electron lasers," Phys. Rev. A, vol. 23, pp. 2537 2552, 1981.

[19] H. P. Freund, P. Sprangle, D. Dillenburg, E. H. daJornada, R. S. Schneider, and B. Liberman, "Collective effects on the operation of 
free-electron lasers with an axial guide field," Phys. Rev. A, vol. 26, pp. 2004-2015, 1982.

[20] H. P. Freund and P. Sprangle, "Unstable electrostatic beam modes in free-electron laser systems," Phys. Rev. A, vol. 27, pp. 1835-1837, 1983.

[21] H. P. Freund, "Nonlinear analysis of free-electron-laser amplifiers with axial guide fields," Phys. Rev. A, vol. 27, pp. 1977-1988, 1983.

[22] P. Sprangle and W. M. Manheimer, "Coherent nonlinear theory of a cyclotron instability," Phys. Fluids, vol. 18, pp. 224-230, 1975

[23] A. Grossman, T. C. Marshall, and S. P. Schlesinger, "A new millimeter free electron laser using a relativistic beam with spiraling electrons," Phys. Fluids, vol. 26, pp. 337-343, 1983.

[24] R. E. Shefer and G. Bekefi, "Cyclotron emission from intense relativistic electron beams in uniform and rippled magnetic fields," Int. J. Electron., vol. 51, pp. 569-582, 1981.

[25] V. L. Bratman, G. G. Denisov, N. S. Ginzburg, and M. I. Petelin, "FEL's with Bragg reflection resonators: Cyclotron autoresonance masers versus ubitrons," IEEE J. Quantum Electron., vol. QE-19, pp. 282-296, 1983.

[26] H. P. Freund, A. K. Ganguly, Y. Y. Lau, and D. Dialetis, private communications.

[27] R. K. Parker, R. H. Jackson, S. H. Gold, H. P. Freund, V. L. Granatstein, P. C. Efthimion, M. Herndon, and A. K. Kinkead, "Axial magnetic-field effects in a collective-interaction free-electron laser at millimeter wavelengths," Phys. Rev. Lett., vol. 48, pp. 238-242, 1982.

[28] S. H. Gold, R. H. Jackson, R. K. Parker, H. P. Freund, V. L. Gran atstein, P. C. Efthimion, M. Herndon, and A. K. Kinkead, "Experimental study of axial magnetic field effects on the operation of a mil limeter-wave free-electron laser," in Physics of Quantum Electronics, Vol. 9: Free-Electron Generators of Coherent Radiation, S. F. Jacobs, G. T. Moore, H. S. Pilloff, M. Sargent, III, M. O. Scully, and R. Spitzer, Eds. Reading, MA: Addison-Wesley, 1982, pp. 741-757.

[29] R. H. Jackson, S. H. Gold, R. K. Parker, H. P. Freund, P. C. Efthimion, V. L. Granatstein, M. Herndon, A.K. Kinkead, J. E. Kosakowski, and T. J. T. Kwan, "Design and operation of a collective millimeter-wave free-electron laser," IEEE J. Quantum Electron., vol. QE-19, pp. 346-356, 1983.

[30] S. C. Chen and T. C. Marshall, "Thomson backscattering from a relativistic electron beam as a diagnostic for parallel velocity spread," Phys. Rev. Lett., vol. 52, pp. 425-428, 1984.

[31] K. L. Felch, L. Vallier, J. M. Buzzi, P. Drossart, H. Boehmer, H. J. Doucet, B. Etlicher, H. Lamain, and C. Rouillé, "Collective freeelectron laser studies," IEEE J. Quantum Electron., vol. QE-17, pp. $1354-1358,1981$.

[32] H. J. Doucet, private communications

[33] J. Masud, T. C. Marshall, and S. P. Schlesinger, "A Raman-FEL amplifier experiment at $1.22 \mathrm{~mm}, "$ Bull. Amer. Phys. Soc., vol. 29, p. 1341,1984

[34] J. Fajans, G. Bekefi, Y. Z. Yin, and B. Lax, "Spectral measurements from a tunable, Raman, free-electron laser," Phys. Rev. Lett., vol. 53, pp. 246-249, 1984.

[35] D. A. Kirkpatrick, R. E. Shefer, and G. Bekefi, "High brightness electrostatically focussed field emission electron guns for free electron laser applications," Massachusetts Inst. Technol., Cambridge, Preprint PFC/JA-84-40, 1984

[36] S. H. Gold, W. M. Black, H. P. Freund, V. L. Granatstein, R. H. Jackson, P. C. Efthimion, and A. K. Kinkead, "Study of gain, bandwidth, and tunability of a millimeter-wave free-electron laser operating in the collective regime," Phys. Fluids, vol. 26, pp. 2683-2688. 1983.

[37] S. H. Gold, W. M. Black, H. P. Freund, V. L. Granatstein, and A. K. Kinkead, "Radiation growth in a millimeter-wave free-electron laser operating in the collective regime," Phys. Fluids, vol. 27, pp. $746-754,1984$

[38] S. H. Gold, W. M. Black, V. L. Granatstein, and A. K. Kinkead, "Breakdown of the atmosphere by emission from a millimeter-wave free-electron maser," Appl. Phys. Lett., vol. 43, pp. 922-924, 1983.

[39] S. H. Gold, W. M. Black, H. P. Freund, V. L. Granatstein, P. C. Efthimion, and A. K. Kinkead, "Studies of radiation growth and emission spectrum of a millimeter-wave free-electron laser operating in the collective regime," in Free-Electron Generators of Coheren Radiation, C. A. Brau, S. F. Jacobs, and M. O. Scully, Eds., Proc. SPIE, vol. 453, pp. 350-355, 1984.

[40] J. A. Pasour and S. P. Schlesinger, "Multichannel grating spectrometer for millimeter waves," Rev. Sci. Instrum., vol. 48, pp. 1355 1356, 1977.

[41] H. P. Freund and A. K. Ganguly, "Electron orbits in free-electron lasers with a helical and an axial guide magnetic field, " this issue, pp. 1073-1079

[42] S. H. Gold, D. L. Hardesty, A. K. Kinkead, L. R. Barnett, and V. L. Granatstein, "High-gain $35-\mathrm{GHz}$ free-electron laser-amplifier experiment," Phys. Rev. Lett., vol. 52, pp. 1218-1221, 1984.

[43] C. W. Roberson, J. A. Pasour, F. Mako, R. F. Lucey, and P. Sprangle, "A free-electron laser driven by a long-pulse induction linac," in Infrared and Millimeter Waves, Vol. 10: Millimeter Components and Techniques, Part II, K. J. Button, Ed. New York: Academic, 1983, pp. 36l-398.

[44] J. A. Pasour, R. F. Lucey, and C. W. Roberson, "Long-pulse freeelectron laser driven by a linear induction accelerator," in Free-Electron Generators of Coherent Radiation, C. A. Brau, S. F. Jacobs, and M. O. Scully, Eds., Proc. SPIE, vol. 453, pp. 328-335, 1984.

145] J. A. Pasour, F. Mako, and C. W. Roberson, "Electron drift in a linear magnetic wiggler with an axial guide field," J. Appl. Phys., vol. 53, pp. 7174-7178, 1982.

[46] J. A. Pasour, R. F. Lucey, and C. A. Kapetanakos, "Long-pulse, high-power, free-electron laser with no external beam focusing," Phys. Rev. Lett., vol. 53, pp. 1728-1731, 1984.

[47] J. E. Leiss, N. J. Norris, and M. A. Wilson, "Design and performance of a long-pulse high-current linear induction accelerator at the National Bureau of Standards," Particle Accelerators, vol. 10, pp. $223-234,1980$

[48] J. J. Ramirez and D. L. Cook, "A study of low-current-density microsecond electron beam diodes," J. Appl. Phys., vol. 5I, pp. 4602$4611,1980$.

[49] J. Fajans, "End effects of a bifilar magnetic wiggler," J. Appl. Phys., vol. 55 , pp. $43-50,1984$.

[50] F. Mako, J. A. Pasour, C. W. Roberson, and R. Lucey, "A broadband, high-power millimeter to centimeter spectrometer," Rev. Sci. Instrum., vol. 55, pp. 712-715, 1984

[51] J. Walsh, private communication.

[52] P. Sprangle, C. M. Tang, and W. M. Manheimer, "Nonlinear formulation and efficiency enhancement of free-electron lasers," Phys. Rev. Lett., vol. 43, pp. 1932-1936, 1979; "Non-linear theory of freeelectron lasers and efficiency enhancement," Phys. Rev. A, vol. 21, pp. $302-318,1980$

[53] L. R. Elias, "High-power, cw, efficient, tunable free-electron laser using low-energy electron beams," Phys. Rev. Lett. , vol. 42, pp. 977981,1979

[54] P. Sprangle and R. A. Smith, "Theory of free-electron lasers," Phys Rev. A, vol. 21, pp. 293-301, 1980.

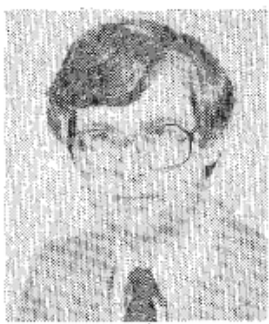

John A. Pasour was born in Gastonia, NC, on July 25,1950 . He received the B.S. and Ph.D. degrees in physic from North Carolina State University, Raleigh, in 1972 and 1977, respectively.

He held a National Research Council Associateship at the Naval Research Laboratory, Washington, DC, from 1977 to 1978. Since 1979, he has been a Research Physicist in the Plasma Physics Division of the Naval Research Laboratory. During that time, his research has dealt with the generation and application of intense electron and ion beams, high-power microwaves, and free-electron lasers. He is currently head of the Long-Pulse Free-Electron Laser Section of the Beam Dynamics Program, where his major interests are high-power single-stage and two-stage FEL oscillators, high-brightness, high-current electron beams and novel radiation sources.

Dr. Pasour is a member of the American Physical Society and Sigma Xi. 


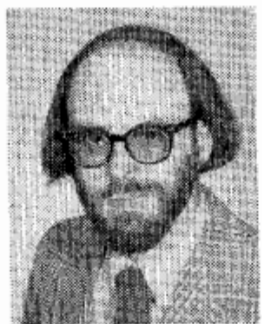

Steven H. Gold was born in Philadelphia, PA, on September 3, 1946. He received the B.A. degree in physics from Haverford College, Haverford, PA, in 1968, the M.S. degree in 1970, and the Ph.D degree in physics in 1978 from the University of Maryland, College Park. His Ph.D. thesis research included an experimental study of plasma turbulence in a high voltage theta pinch by means of collective laser scattering techniques.

From 1978 to 1980 , he held a National Research Council Resident Research Associateship with the Laser Plasma Branch of the Naval Research Laboratory, Washing-

ton, DC. During this period, he studied energy transport through laseraccelerated thin-foil targets using optical diagnostic techniques. Since 1980. he has held the position of Research Physicist with the High Power Electromagnetic Radiation Branch of the Naval Research Laboratory. He is presently studying the generation of very high power coherent millimeter-wave radiation from intense relativistic electron beams in oscillator, amplifier, and superradiant configurations by means of the free-electron laser and cyclotron maser interactions.

Dr. Gold is a member of the Plasma Physics Division of the American Physical Society, of the Society of America and its National Capital Section, and of the American Association for the Advancement of Science. 